University of Wollongong

Research Online

Faculty of Engineering and Information

Faculty of Engineering and Information

Sciences - Papers: Part B

Sciences

2018

Experimental study on simultaneously measuring Young's modulus and internal fraction using self-mixing system

\author{
Fei Xia \\ University of Wollongong, fx999@uowmail.edu.au \\ Bin Liu \\ University of Wollongong, bl987@uowmail.edu.au \\ Lingzhi Cao \\ Zhengzhou University of Light Industry \\ Yanguang Yu \\ University of Wollongong, yanguang@uow.edu.au \\ Jiangtao Xi \\ University of Wollongong, jiangtao@uow.edu.au
}

See next page for additional authors

Follow this and additional works at: https://ro.uow.edu.au/eispapers1

Part of the Engineering Commons, and the Science and Technology Studies Commons

Research Online is the open access institutional repository for the University of Wollongong. For further information contact the UOW Library: research-pubs@uow.edu.au 


\title{
Experimental study on simultaneously measuring Young's modulus and internal fraction using self-mixing system
}

\author{
Abstract \\ Material parameters such as Young's modulus and internal friction are important for estimation of \\ material performance. This paper presents an experimental study for measuring material related \\ parameters using a selfmixing interferometric (SMI) configuration. An SMI system consists of a laser \\ diode (LD), a lens and an external target to be measured. When a part of the lasing light back-reflected or \\ back-scattered by the external target re-enters the LD internal cavity, both optical frequency and intensity \\ of the lasing light can be modulated. This modulated laser intensity is often referred as SMI signal. \\ Generally, the target related movement or its surface information can be retrieved from this SMI signal. In \\ this paper, an SMI system is implemented. A tested sample is used as the target to form the external \\ cavity of the LD. The tested sample is stimulated in vibration. Continuous wavelet transform (CWT) is \\ utilized to retrieve the vibration information of the tested sample from an SMI signal. We are able to \\ obtain both Young's modulus and internal friction from a piece of an experimental SMI signal. This work \\ provides a novel, simple non-destructive solution for simultaneous measurement of Young's modulus and \\ internal friction.

\section{Disciplines} \\ Engineering | Science and Technology Studies

\section{Publication Details} \\ F. Xia, B. Liu, L. Cao, Y. Yu, J. Xi, Q. Guo \& J. Tong, "Experimental study on simultaneously measuring \\ Young's modulus and internal fraction using self-mixing system," in Semiconductor Lasers and \\ Applications VIII, 2018, pp. 1081212-1-1081212-9.
}

\section{Authors}

Fei Xia, Bin Liu, Lingzhi Cao, Yanguang Yu, Jiangtao Xi, Qinghua Guo, and Jun Tong 


\section{Experimental study on simultaneously measuring Young's modulus and internal fraction using self-mixing system}

Fei Xia, Bin Liu, Lingzhi Cao, Yanguang Yu, Jiangtao $\mathrm{Xi}$, et al.

Fei Xia, Bin Liu, Lingzhi Cao, Yanguang Yu, Jiangtao Xi, Qinghua Guo, Jun Tong, "Experimental study on simultaneously measuring Young's modulus and internal fraction using self-mixing system," Proc. SPIE 10812, Semiconductor Lasers and Applications VIII, 1081212 (6 November 2018); doi: $10.1117 / 12.2500741$ 


\title{
Experimental study on simultaneously measuring Young's modulus and internal fraction using self-mixing system
}

\author{
Fei Xia ${ }^{\mathrm{a}}$, Bin Liu ${ }^{\mathrm{a}}$, Lingzhi Cao*b ${ }^{\mathrm{b}}$, Yanguang $\mathrm{Yu}^{\mathrm{a}}$, Jiangtao $\mathrm{Xi}^{\mathrm{a}}$, Qinghua Guo ${ }^{\mathrm{a}}$, Jun Tong ${ }^{\mathrm{a}}$ \\ ${ }^{a}$ School of Electrical, Computer and Telecommunications Engineering, University of \\ Wollongong, Wollongong, NSW, 2522, Australia; ${ }^{\mathrm{b}}$ School of Electrical and Information \\ Engineering, Zhengzhou University of Light Industry, Zhengzhou, Henan, 450000, China
}

\begin{abstract}
Material parameters such as Young's modulus and internal friction are important for estimation of material performance. This paper presents an experimental study for measuring material related parameters using a selfmixing interferometric (SMI) configuration. An SMI system consists of a laser diode (LD), a lens and an external target to be measured. When a part of the lasing light back-reflected or back-scattered by the external target re-enters the LD internal cavity, both optical frequency and intensity of the lasing light can be modulated. This modulated laser intensity is often referred as SMI signal. Generally, the target related movement or its surface information can be retrieved from this SMI signal. In this paper, an SMI system is implemented. A tested sample is used as the target to form the external cavity of the LD. The tested sample is stimulated in vibration. Continuous wavelet transform (CWT) is utilized to retrieve the vibration information of the tested sample from an SMI signal. We are able to obtain both Young's modulus and internal friction from a piece of an experimental SMI signal. This work provides a novel, simple non-destructive solution for simultaneous measurement of Young's modulus and internal friction.
\end{abstract}

Keywords: self-mixing interferometry, laser diode, Young's modulus, internal friction, wavelet transform

\section{INTRODUCTION}

Material related parameters such as Young's modulus and internal friction are important for mechanical and material engineering. These parameters play key roles in the material performances. It has been a great interest to measure the value of these parameters. Traditional methods including tensile test [1], flexure test [2], and others $[3,4]$ are static methods which are often conducted based on the direct measurement of the stresses and strains of the tested sample loaded by a known force. As a result, the tested sample is often damaged after the test, as a relatively high stress must be applied to reach a measurable deformation. Therefore, more interests have been focusing on the dynamic methods, which are non-destructive and more accurate with a simpler system.

In recent years, the impulse excitation technique (IET), a non-destructive technique to determine Young's modulus and internal friction of the material has attracted great attention. The detection system used for IET is normally microphone, accelerometer and so on. Self-mixing interferometry (SMI), an emerging sensing technique, which is non-destructive and non-contact has been developed for high accuracy sensing applications, such as displacement [5], velocity [6] and distance [7] measurement and so on. It is also suitable for the material related parameters measurement. Figure 1 shows a basic SMI system, which consists of a LD and a target to form the external cavity of the LD. When a part of the lasing light emitted by the LD back-reflects or backscatters by the external target and re-enters the LD internal cavity, both optical frequency and the intensity of the lasing light can be modulated. This modulated laser intensity contains the vibration information of the external target.

Semiconductor Lasers and Applications VIII, edited by Ning Hua Zhu, Werner H. Hofmann, Proc. of SPIE Vol. 10812, 1081212 - @ 2018 SPIE · CCC code: 0277-786X/18/\$18 · doi: 10.1117/12.2500741 


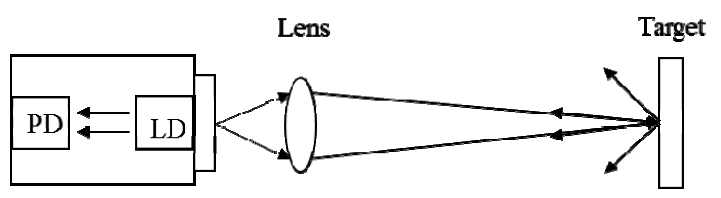

Figure 1. Basic SMI system

This paper proposes an intact system design for Young's modulus and internal friction measurement based on SMI technique with IET. An experimental SMI system is built, where a tested sample for the experiments is used as the external target. With a light strike on the tested sample, the tested sample is stimulated into damping vibration. This damping vibration signal is captured by the SMI system and a corresponding SMI signal is generated. By applying wavelet transform onto the SMI signal, both fundamental resonant frequency and the magnitude decay of the damping vibration from the tested sample can be obtained, from which we can calculate material related parameters Young's modulus and internal friction.

\section{MEASUREMENT PRINCIPLE}

The widely accepted SMI model is derived from the Lang Kobayashi equations [8, 9]. The SMI model is relisted below to investigate the relationship between Young's modulus and internal friction and the SMI signal.

$$
\begin{gathered}
\phi_{0}=4 \pi L / \lambda \\
\phi_{s}=\phi_{0}-C \sin \left(\phi_{s}+\arctan \alpha\right) \\
G(t)=\cos \left(\phi_{s}\right) \\
P(t)=P_{0}[1+m G(t)]
\end{gathered}
$$

where the physical meaning of each parameter is described as following in the Table 1.

Table 1. Meanings of the symbols in the L-K equations

\begin{tabular}{ll}
\hline Symbols & Physical meaning \\
$\phi_{0}$ & light phase without external optical feedback \\
$L$ & Distance between the LD front facet and the external target \\
$\lambda$ & Laser wavelength \\
$\phi_{S}$ & Light phase with external optical feedback \\
$C$ & Optical feedback factor \\
$\alpha$ & Linewidth enhancement factor \\
$G(t)$ & Interferometric function \\
$P(t)$ & Laser power emitted by the LD with external optical feedback \\
$P_{0}$ & Laser power emitted by the LD without external optical feedback \\
$m$ & Modulation index \\
\hline
\end{tabular}

Generally, we treat $G(t)$ as the SMI signal as it can be normalized from the laser power $P(t)$. In our system, the external target is the tested sample, so that $L=L_{0}+y(t)$, where $L_{0}$ is the initial external cavity length, that is the distance between the LD and the tested sample in static state, $y(t)$ is the damping vibration of the tested sample. Based on the equation of motion [10], the damping vibration $y(t)$ can be written as

$$
y(t)=A_{0} e^{-k t} \cos \left(2 \pi f_{R O} t\right)
$$

Where $A_{0}$ is the maximum amplitude of the damping vibration, $k$ is the damping factor, $f_{R O}$ describes the resonant frequency of the tested sample. The Young's modulus $E$ of the tested sample can be calculated as [11] 


$$
E=0.9465 \cdot \frac{m f_{R O}^{2} L^{3}}{b h^{3}} \cdot T
$$

where $T=1+6.585\left(\frac{h}{L}\right)^{2}$, when $L / h \geq 20$ [12]. $m, L, b$, and $h$ are the mass, length, width, and height of the tested sample, which can be determined from the dimension of the tested sample. The remaining value needs to be determined is $f_{R O}$, which can be found in equation (5). The internal friction $Q^{-1}$ of the tested sample can be calculated as [13]

$$
Q^{-1}=\frac{k}{\pi f_{R O}}
$$

where the values need to be determined are $k$ and $f_{R O}$, which can be found in equation (5). The Young's modulus $E$ and the internal friction $Q^{-1}$ can be calculated by knowing information in the damping vibration of the tested sample $y(t)$, which can be determined from the SMI signal $G(t)$.

The simulation is conducted to investigate the relationship between the SMI signal $G(t)$ and the damping vibration of the tested sample $y(t)$. For the SMI part, we assume the laser wavelength $\lambda=7.85 \times 10^{-7} \mathrm{~m}$, the initial external cavity length $L_{0}=0.1 \mathrm{~m}$, the optical feedback $C=3$ and the linewidth enhancement factor $\alpha=6$. The sampling frequency is $100 \mathrm{kHz}$. For the IET part, we assume the maximum amplitude of the damping vibration $A_{0}=4.317 \times 10^{-6} \mathrm{~m}$, the damping factor $k=4.6$ and the resonant frequency $f_{R O}=444 \mathrm{~Hz}$. We can write the damping vibration as $y(t)=4.317 \times 10^{-6} e^{-4.6 t} \cos (2 \pi(444))$. The simulated damping vibration and its corresponding SMI signal $G(t)$ are shown in Figure 2. From Figure 2 we can observe that, the fundamental period of SMI signal $G(t)$ is the same as the period of the damping vibration signal $y(t)$. The fringe numbers on the SMI signal $G(t)$ indicates the magnitude of the damping vibration in each vibration period, where the fringe numbers decrease as the damping vibration magnitude decreases with time. It can be found that the information of the damping vibration $y(t)$, which are the resonant frequency $f_{R O}$ and the damping factor $k$, are carried in the SMI signal $G(t)$.
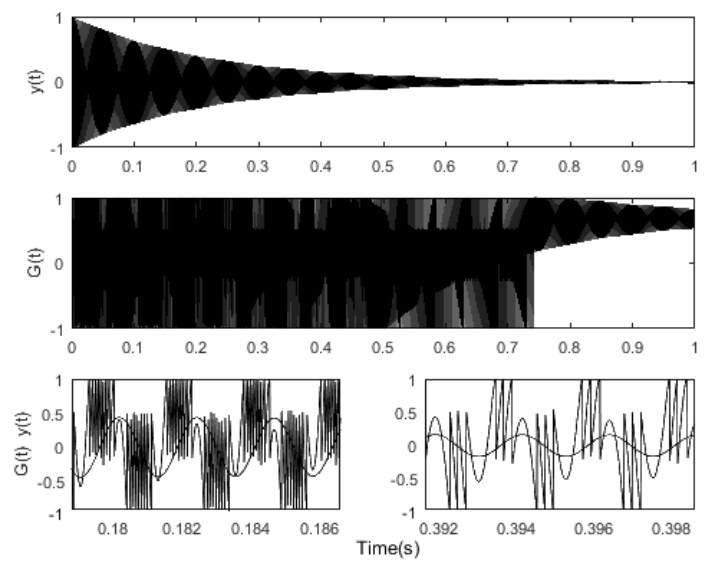

Figure 2. Simulated damping vibration and its corresponding SMI signal

The resonant frequency $f_{R O}$ are the dominating frequency in the SMI signal and the damping factor $k$ are indicated as the high frequency decay in the SMI signal $G(t)$. Both information can be found in the frequency 
domain of the SMI signal. To achieve this, the continuous wavelet transform (CWT) can be applied onto the SMI signal $G(t)$.

\section{EXPERIMENT STUDY}

\subsection{Experimental setup}

The experiment work is conducted on a brass bar with the mass $m=29.84 \mathrm{~g}$, length $L=138.10 \mathrm{~mm}$, width $b=12.01 \mathrm{~mm}$, and height $h=2.08 \mathrm{~mm}$. The overall experiment setup is shown in figure 3 . The experiment setup consists of two parts: IET part and SMI part. In the IET part, it consists of a tested sample, a stimulate tool, and the support. The stimulate tool is used to strike the tested sample and a damping vibration can be generated. In the SMI part, it consists of a LD (Thorlabs, RLD65MZT2), a photodiode (PD) packaged in the rear of the LD, a temperature (Thorlabs, TED200C), a LD controller (Thorlabs, LDC200C), a trans-impedance amplifier, an oscilloscope, a DAQ card (NI USB-6361) and a personal computer. A SMI signal is be generated corresponding to the damping vibration and picked up by the PD. The temperature controller and LD controller are used to ensure a stable condition of the LD. The trans-impedance amplifier is to transfer the current signal from the PD into a voltage signal and have it amplified. A DAQ card is used to sample the signal The sampled SMI signal isthen displayed on a personal computer. The amplified signal can be also displayed on an oscilloscope. The SMI signal is normally a saw tooth-like waveform, by doing signal processing onto the SMI signal, we can retrieve the material related parameters such as Young's modulus and internal friction.

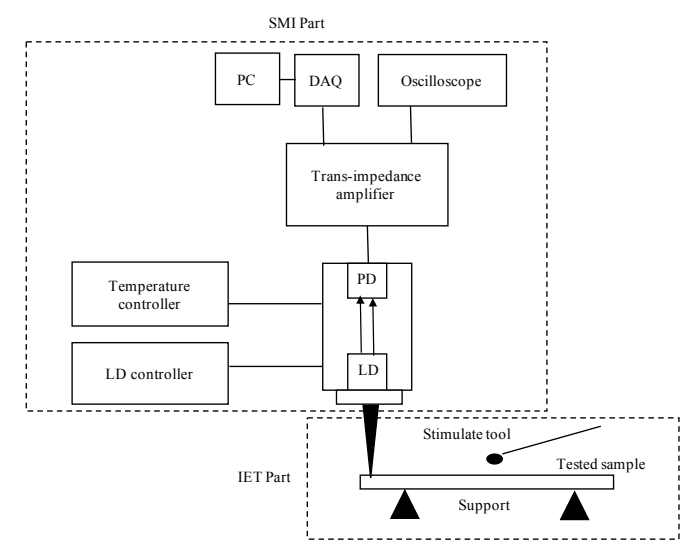

Figure 3. Overall experiment setup

\subsection{Experimental results}

Figure 4 shows the experimental SMI signal from the system. The laser current and the temperature are set to be $14 \mathrm{~mA}$ and $25^{\circ} \mathrm{C}$. The sampling rate is $200 \mathrm{kHz}$ and the data length of a SMI signal is $1 \mathrm{~s}$. 

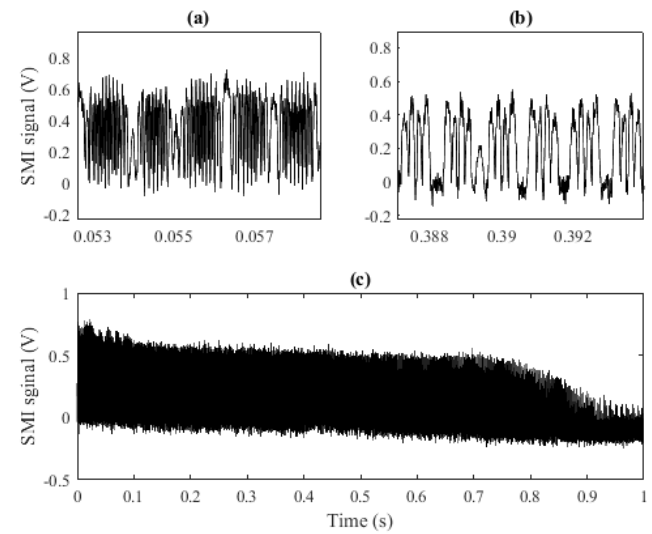

Figure 4. Experimental SMI signal. (a), (b) zoomed in parts of (c) SMI signal

In figure 4, the top two figures (a) and (b) are the zoomed in parts of the SMI signal. It is clear to see that the fringe numbers decrease with the time. The continuous wavelet transform (CWT) is applied onto the SMI signal to retrieve the material related parameters. The CWT is a method to investigate the frequency domain of a signal, especially when frequency content varies over time [14]. The CWT of a SMI signal can be written as

$$
W(a, b)=\frac{1}{\sqrt{a}} \int_{-\infty}^{+\infty} G(t) \psi\left(\frac{t-b}{a}\right) d t
$$

where $W(a, b)$ is the coefficient of the transform, $a$ represents the scale wavelet parameter, $b$ represents the shifting wavelet parameter and $\psi\left(\frac{t-b}{a}\right)$ is the scaled and shifted version of mother wavelet $\psi(t) . W(a, b)$ is normalized by $\frac{1}{\sqrt{a}}$ to ensure all the wavelets have the same energy at every scale. Scale $a$ is inversely proportional to the frequency component of the analyzed signal. There are different mother wavelets, the choosing of one of them depends on the nature of the analysed signal. The Morlet complex wavelet is chosen to analyse our SMI signals because it shows good results in analysing sinusoidal-like signal [15] and an SMI signal waveform is sinusoidal-like or sawtooth-like.. The Morlet wavelet is defined in the time domain as

$$
\psi(t)=e^{i \omega_{e} t} e^{\frac{-t^{2}}{2}}
$$

In the frequency domain

$$
\Psi(\omega)=\int_{-\infty}^{+\infty} \psi(t) e^{-j \omega t} d t=\sqrt{2 \pi} e^{\frac{-\left(\omega-\omega_{e}\right)^{2}}{2}}
$$

Where $\omega_{e}$ is the central angular frequency of the wavelet. The admissibility condition for a wavelet is

$$
\int_{0}^{+\infty} \frac{|\Psi(\omega)|^{2}}{|\omega|} d \omega<+\infty
$$

It requires the Fourier transform of the wavelet should be zero at zero frequency, i.e. $|\Psi(\omega)|=0$ when $\omega=0$. Using the admissibility condition in equation (11), the following can be obtained 


$$
\Psi(0)=\sqrt{2 \pi} e^{\frac{-\omega_{e}^{2}}{2}}
$$

Figure 5 shows the value of $\Psi(0)$ at different $\omega_{e}$ for Morlet wavelet. For $\omega_{e} \geq 5$, the admissibility condition is fulfilled. $\omega_{e}=6$ is chosen for the simulation.

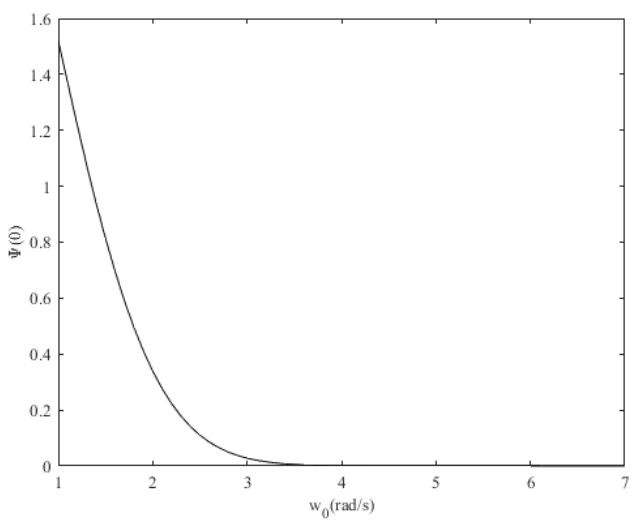

Figure 5. Admissibility condition for the Morlet wavelet

In wavelet analysis, the way to relate scale parameter to frequency is to determine the central frequency of the wavelet, $f_{e}=\omega_{e} /(2 \pi)$, where we have set the value for $\omega_{e}=6$. So $f_{e}=\omega_{e} /(2 \pi) \approx 0.9549 H z$. The frequency content (denoted by $f_{a}$ ) is $f_{a}=\frac{f_{e}}{a}=\frac{0.9549}{a}$. Due to the dyadic nature of wavelets [16], it is computationally efficient to choose scale parameter $a$ in terms of a power of $2 . a=a_{0} \times 2^{j d}, j=0,1,2 . . J-1$, where $a_{0}$ is the smallest resolvable scale corresponding to the Nyquist frequency which is half of the sampling frequency $f_{\max }=\frac{f_{s}}{2}=\frac{100 \mathrm{kHz}}{2}=50 \mathrm{kHz}$ (the sampling frequency $f_{s}$ was set as $100 \mathrm{kHz}$ for the sampling rate is $200 \mathrm{kHz}$ ). Then we have $a_{0}=\frac{f_{0}}{f_{\max }}=\frac{0.9549}{50 \mathrm{kHz}} \cdot d$ is the increment in scale and it is chosen to be $1 / 16$ to balance the resolution and computing time. The total scale $J$ can be set based on the frequency range to be analysed, where we already have the maximum frequency $f_{\max }=50 \mathrm{kHz}$, and we set the minimum frequency to be $f_{\text {min }}=1 \mathrm{~Hz}$. Therefore, we will analyze the frequency range between $1 \mathrm{~Hz}$ and $5 \times 10^{4} \mathrm{~Hz}$ which determines the number of octaves $N=$ floor $\left(\log _{2}\left(\frac{f_{\max }}{f_{\min }}\right)\right)=15$. The total number of scales are $J+1=\frac{N}{d}+1=241$.

Compute the Morlet wavelet CWT of the experimental SMI signal $G(t)$, that is $W(a, b)$ by equation (8). The scalogram of the real part of $W(a, b)$ is plotted to visualize the spatial and temporal components of experimental SMI signal $G(t)$, as shown in Figure 6 


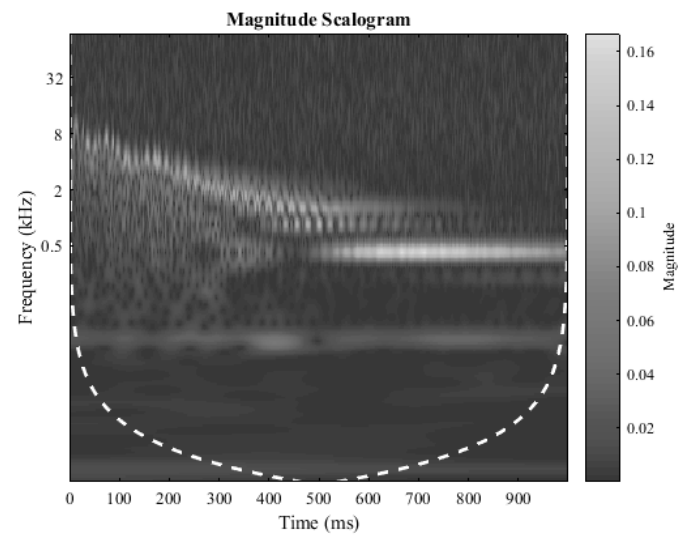

Figure 6. Scalogram of the CWT of experimental SMI signal

In Figure 6, the horizontal axis represents the time index, the vertical axis represents the frequency index (note that the vertical axis is logarithmic) and the colour means the magnitude (from 0.02 to 0.16 ). It can be seen that the yellow colour located at frequency around $500 \mathrm{~Hz}$ appears in the time period from $t=500 \mathrm{~ms}$ to about $t=900 \mathrm{~ms}$, which is the dominating frequency contained in the experimental SMI signal $G(t)$. It is the same as the frequency of the target vibration. It also can be found that a yellow line in decreasing trend from $t=0 \mathrm{~ms}$ to about $t=500 \mathrm{~ms}$ with frequency varying from $8 \mathrm{kHz}$ to $1 \mathrm{kHz}$. This decreasing trend line indicates the decreasing of the fringe numbers in each vibration period in the experimental SMI signal $G(t)$.

Firstly, we determine the value of the dominating frequency by extracting the frequency component with maximum magnitude from the scalogram in Figure 6. The frequency component with maximum magnitude is $f=424.8 \mathrm{~Hz}$, which is shown in Figure 7.

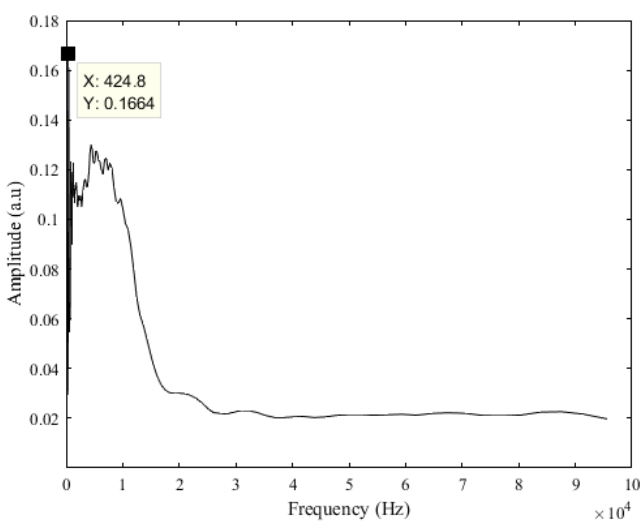

Figure 7. Real part $W(a, b)$ plotted against the frequency, at $\mathrm{Hz}$, denoting the maximum frequency is $443.6 \mathrm{~Hz}$

Then, we determine the value of the slope of the decreasing trend., In order to do so, we need to locate the high frequency component from the scalogram in Figure 6. That can be achieved by extracting the frequency component with maximum magnitude at each time instance. Figure 8 (a) shows the frequency component with maximum magnitude at each time instance, Figure 8 (b) is the curve-fitting result of Figure 8 (a). From the curve-fitting result, it can be seen that most of the points fall on the curve, which shows that the curve can represent the decent trend of the frequency. The decent slope of the curve is $-2.30 \times 10^{-5}$. The sampling rates are $200 \mathrm{kHz}$ so the measured damping factor is $k=-4.6$. 

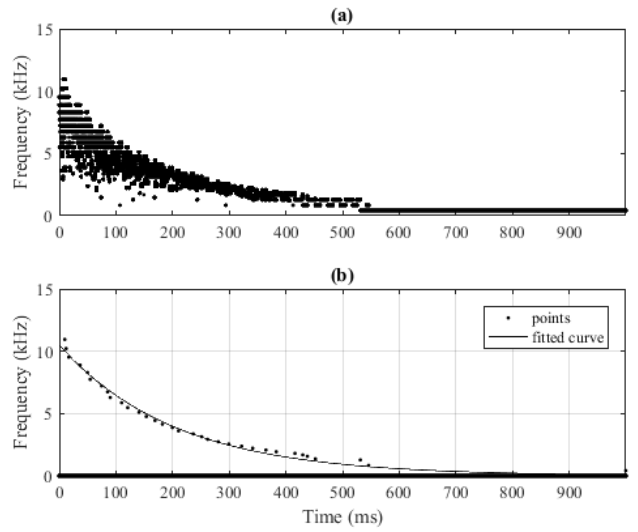

Figure 8. (a) Frequency with maximum amplitude against time. (b) curve-fitting result after excluding other points

The measured resonant frequency is $f=424.8 \mathrm{~Hz}$ and damping factor is $k=-4.6$. Based on the equations (6) and (7), the Young's modulus and internal friction can be calculated. The experiments are repeated 10 times with the brass sample. The average measured value and the standard deviation $(\sigma)$ is shown in Table 2 .

Table 2. Measurement results of brass sample

\begin{tabular}{|c|c|c|c|c|}
\hline $\begin{array}{c}\text { Resonant frequency } \\
f(\mathrm{~Hz})\end{array}$ & $\begin{array}{c}\text { Damping factor } \\
k\end{array}$ & $\begin{array}{c}\text { Young's modulus } \\
E(\mathrm{GPa})\end{array}$ & $\begin{array}{c}\text { Internal friction } \\
Q^{-1}\end{array}$ & $\begin{array}{c}\text { Standard deviation } \\
\sigma\end{array}$ \\
\hline 424.8 & -4.6 & 120.9 & $3.4 \times 10^{-3}$ & 0.03 \\
\hline
\end{tabular}

The standard deviation $\sigma$ is obtained by $\sigma=\sqrt{\frac{1}{N} \sum_{i=1}^{N}\left(x_{i}-\mu\right)^{2}}$, where $N=20, x_{i}$ refers to each measured value, and $\mu$ is the mean value over the measured 10 values. The measured value of Young's modulus for brass sample is also very close value between $102 \mathrm{GPa}$ and $125 \mathrm{GPa}$ reported in the literature [17].

\section{CONCLUSION}

This paper proposes an intact system design for simultaneous measurement of Young's modulus and internal friction. An experimental system has been built and SMI signals have been captured. By applying wavelet transform onto the SMI signal, the material related parameters, such as resonant frequency and damping factor are retrieved simultaneously, so that to achieve the measurements of Young's modulus and internal friction of the tested sample. The experimental results prove the feasibility of using SMI technique for non-destructive and real-time measurement of material related parameters.

\section{REFERENCES}

[1] B. Demczyk et al., "Direct mechanical measurement of the tensile strength and elastic modulus of multiwalled carbon nanotubes," Materials Science and Engineering: A, vol. 334, no. 1-2, pp. 173-178, 2002.

[2] D. K. Shetty, A. R. Rosenfield, P. McGuire, G. K. Bansal, and W. H. Duckworth, "Biaxial flexure tests for ceramics," American Ceramic Society Bulletin, vol. 59, no. 12, pp. 1193-1197, 1980.

[3] P. DeHoff, K. Anusavice, and P. Hathcock, "Materials Science: An Evaluation of the Four-point Flexural Test for Metal-Ceramic Bond Strength," Journal of dental research, vol. 61, no. 9, pp. 1066$1069,1982$. 
[4] A. Bhattacharya and W. Nix, "Analysis of elastic and plastic deformation associated with indentation testing of thin films on substrates," International Journal of Solids and Structures, vol. 24, no. 12, pp. 1287-1298, 1988.

[5] M. Wang and G. Lai, "Displacement measurement based on Fourier transform method with external laser cavity modulation," Review of Scientific Instruments, vol. 72, no. 8, pp. 3440-3445, 2001.

[6] L. Scalise, Y. Yu, G. Giuliani, G. Plantier, and T. Bosch, "Self-mixing laser diode velocimetry: application to vibration and velocity measurement," IEEE Transactions on Instrumentation and Measurement, vol. 53, no. 1, pp. 223-232, 2004.

[7] Z. Duan, Y. Yu, B. Gao, and C. Jiang, "Absolute distance measurement based on multiple self-mixing interferometry," Optics Communications, vol. 389, no. Supplement C, pp. 270-274, 2017/04/15/ 2017.

[8] R. Lang and K. Kobayashi, "External optical feedback effects on semiconductor injection laser properties," IEEE journal of Quantum Electronics, vol. 16, no. 3, pp. 347-355, 1980.

[9] Y. Yu, J. Xi, and J. F. Chicharo, "Measuring the feedback parameter of a semiconductor laser with external optical feedback," Optics express, vol. 19, no. 10, pp. 9582-9593, 2011.

[10] S. B. Coşkun, B. Öztürk, and M. T. Atay, Transverse vibration analysis of euler-bernoulli beams using analytical approximate techniques. INTECH Open Access Publisher, 2011.

[11] E. ASTM, "Standard test method for dynamic young's modulus, shear modulus, and poisson's ratio by impulse excitation of vibration," Annual Book of ASTM Standards, vol. 3, 1876.

[12] S. Spinner, T. Reichard, and W. Tefft, "A comparison of experimental and theoretical relations between Young's modulus and the flexural and longitudinal resonance frequencies of uniform bars," $J$. Res. Natl. Bur. Stand., Sect. A, vol. 64, pp. 147-155, 1960.

[13] G. Roebben, B. Bollen, A. Brebels, J. Van Humbeeck, and O. Van der Biest, "Impulse excitation apparatus to measure resonant frequencies, elastic moduli, and internal friction at room and high temperature," Review of scientific instruments, vol. 68, no. 12, pp. 4511-4515, 1997.

[14] W. Staszewski, "Identification of damping in MDOF systems using time-scale decomposition," Journal of sound and vibration, vol. 203, no. 2, pp. 283-305, 1997.

[15] W. K. Ngui, M. S. Leong, L. M. Hee, and A. M. Abdelrhman, "Wavelet analysis: Mother wavelet selection methods," in Applied mechanics and materials, 2013, vol. 393, pp. 953-958: Trans Tech Publ.

[16] D. S. G. Pollock, "Dyadic Wavelets Analysis."

[17] R. M. Digilov and H. Abramovich, "Flexural vibration test of a beam elastically restrained at one end: a new approach for Young's modulus determination," Advances in Materials Science and Engineering, vol. 2013, 2013. 